\title{
Synthesis and Spectral Studies of Novel Diazepine Derivatives and Study in Specific Reference to Tautomerization
}

\author{
R. K. SAINI ${ }^{\mathrm{a}}$, Y. C. JOSHI ${ }^{\mathrm{a}}$ and P. JOSHI ${ }^{\mathrm{b}}$ \\ ${ }^{a}$ Department of Chemistry, University of Rajasthan, Jaipur-302004, India \\ ${ }^{\mathrm{b}} \mathrm{S}$. S. Jain Subodh P. G. College, Jaipur, Rajasthan, India \\ sainirk97@yahoo.co.in
}

Received 10 December 2012 / Accepted 12 January 2013

\begin{abstract}
Condensation of various 1,3-diketone derivatives (1a-1f) with ethylene diamine and O-PDA in absolute ethanol led to synthesis of new 1,4-diazepines and 1,5-benzodiazepine derivatives. Structure of newly synthesized 1,4-diazepines (2a-2f) and 1,5-benzodiazepine derivatives (3a-3f) were established on the spectral studies viz: IR, ${ }^{1} \mathrm{H}$ NMR, ${ }^{13} \mathrm{C}$ NMR.
\end{abstract}

Keywords: 1,3-Diketone, Ethylene diamine, O-PDA, Diazepine, Benzodiazepine

\section{Introduction}

Diazepine and its derivatives are important class of compounds possess various biological activities viz: anticancer ${ }^{1}$, antibacterial ${ }^{2}$, antiemetic ${ }^{3}$, anticonvulsant ${ }^{4}$, fungicidal, insecticidal and herbicidal $^{5}$, antiviral ${ }^{6}$, antihypertensive ${ }^{7}$, antidepressant ${ }^{8}$, antiasthamatic ${ }^{9}$, anti-inflammatory agent ${ }^{10}$.Benzodiazepines are also used in elderly as community dwelling population ${ }^{11}$.

Interestingly, benzothienobenzodiazepine(Y-931), dibenzo [b,f]Diazepine (clozapine) and thienobenzodiazepine (Olanzapine) are known as typical effectiveantipsychotics ${ }^{12,13}$. Naturally occurring benzodiazepines, such as pyrrolo [2, 1-e] 1, 4-benzodiazepines (PBDs) isolated from streptomyces species ${ }^{14}$ are found effective as antitumour ${ }^{15}$, antibiotics and in DNA probe ${ }^{16}$. Realizing the medicinal important of diazepines derivatives and in continuation of our earlier work $^{17}$, in this paper we report the synthesis of some new 1, 4-diazepines and 1, 5-benzodiazepine derivatives.

\section{Experimental}

Melting points were uncorrected. The IR spectra were recorded in $\mathrm{KBr}$ disks on NicoletMegna-FT-IR550 spectrometers. ${ }^{1} \mathrm{H}$ NMR and ${ }^{13} \mathrm{C}$ NMR recorded on model DRX 300 at $300.13 \& 75.48 \mathrm{MHz}$ respectively in $\mathrm{CDCl}_{3} \mathrm{DDMSO}_{\mathrm{d}} \mathrm{d}_{6}$ using TMS as internal standard. The purity of newly synthesized compounds was checked by TLC.

Generalized preparation of diazepine nucleus (2a)

A mixture of diketones (1a-1f, $0.01 \mathrm{M})$ and ethylenediamine $(0.01 \mathrm{M})$ were refluxed in absolute ethanol $(10 \mathrm{~mL})$ by making reaction medium slightly acidic. The reaction mixture was 
refluxed for 3 hours and then cooled to room temperature. The solid residue was recrystallized from acetone-ethanol mixture yield crystalline product. Purity of compound were checked by TLC using (benzene: ethanol: ammonia: 7: 2:1) upper layer as mobile phase.

\section{Generalized preparation of benzodiazepine nucleus (3a)}

A mixture of diketones (1a-1f, $0.01 \mathrm{M})$ and O-PDA $(0.01 \mathrm{M})$ were refluxed in absolute ethanol $(10 \mathrm{~mL})$ by making reaction medium slightly acidic. The reaction mixture was refluxed for 6-8 hours and then cooled to room temperature. The solid residue was recrystallized from ethanol yield crystalline product. Purity of compound were checked by TLC using (benzene: ethanol: ammonia: 7: 2: 1) upper layer as mobile phase.

\section{Results and Discussion}

Condensation of propane-1-(1,3-benzodioxol-5yl)-2-(quinoline-8-sulfonyl)-3-phenyl-1,3dione (1a) or other compounds(1b-1f) having various substituent in phenyl ring, with ethylene diamine in absolute ethanol and refluxing for 3 hours results in the formation of 5-substituted phenyl-6-(quinoline-8-sulfonyl)-7-(1,3-benzodioxol-5yl)-6H-2,3-dihydro-1,4-diazepine.

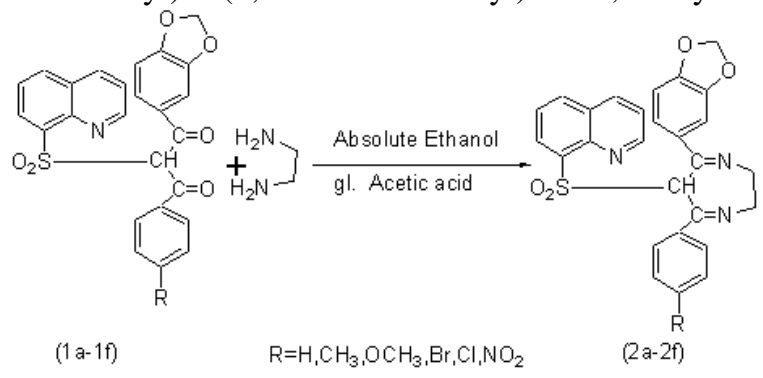

\section{Scheme 1}

Similarly condensation of propane-1-(1,3-benzodioxol-5yl)-2-(quinoline-8-sulfonyl)-3phenyl-1,3-dione(1a) or other compounds(1b-1f) having various substituent in phenyl ring, with ethylene diamine in absolute ethanol and refluxing for 6-8 hours results in the formation of 2-substituted elemental analysis and spectral analysis of title compounds are given in Tables $1 \& 2$ respectively.

Table 1. Elemental analysis of title compounds

\begin{tabular}{cccccccc}
\hline \multirow{2}{*}{ Compd. } & Molecular & M.P & Yields & \multicolumn{4}{c}{ Elemental analysis calculated (found) } \\
\cline { 5 - 8 } & formula & ${ }^{0} \mathrm{C}$ & $\%$ & $\mathrm{C}$ & $\mathrm{H}$ & $\mathrm{N}$ & $\mathrm{S}$ \\
\hline $\mathbf{2 a}$ & $\mathrm{C}_{27} \mathrm{H}_{21} \mathrm{O}_{4} \mathrm{~N}_{3} \mathrm{~S}$ & 187 & 40 & $67.08(67.05)$ & $4.34(4.32)$ & $8.69(8.50)$ & $6.62(6.60)$ \\
2b & $\mathrm{C}_{28} \mathrm{H}_{23} \mathrm{O}_{4} \mathrm{~N}_{3} \mathrm{~S}$ & 176 & 35 & $67.60(67.50)$ & $4.62(4.50)$ & $8.45(8.43)$ & $6.43(6.40)$ \\
2c & $\mathrm{C}_{28} \mathrm{H}_{23} \mathrm{O}_{5} \mathrm{~N}_{3} \mathrm{~S}$ & 150 & 42 & $65.49(65.40)$ & $4.48(4.40)$ & $8.18(8.10)$ & $6.23(6.21)$ \\
2d & $\mathrm{C}_{27} \mathrm{H}_{20} \mathrm{O}_{4} \mathrm{~N}_{3} \mathrm{SBr}$ & $\mathrm{Oily}$ & 47 & $57.70(57.30)$ & $3.56(3.52)$ & $7.47(7.40)$ & $5.69(5.62)$ \\
2e & $\mathrm{C}_{27} \mathrm{H}_{20} \mathrm{O}_{4} \mathrm{~N}_{3} \mathrm{SCl}$ & 210 & 37 & $62.60(62.75)$ & $3.86(3.83)$ & $8.11(8.03)$ & $6.18(6.10)$ \\
2f & $\mathrm{C}_{27} \mathrm{H}_{20} \mathrm{O}_{6} \mathrm{~N}_{4} \mathrm{~S}$ & $\mathrm{Oily}$ & 30 & $61.36(61.25)$ & $3.78(3.70)$ & $10.60(10.5)$ & $6.06(6.01)$ \\
3a & $\mathrm{C}_{31} \mathrm{H}_{21} \mathrm{O}_{4} \mathrm{~N}_{3} \mathrm{~S}$ & 193 & 50 & $70.05(70.01)$ & $3.76(3.70)$ & $7.90(7.87)$ & $6.02(6.11)$ \\
3b & $\mathrm{C}_{32} \mathrm{H}_{23} \mathrm{O}_{4} \mathrm{~N}_{3} \mathrm{~S}$ & 140 & 55 & $70.45(70.62)$ & $4.72(4.65)$ & $7.70(7.65)$ & $5.87(5.81)$ \\
3c & $\mathrm{C}_{32} \mathrm{H}_{23} \mathrm{O}_{5} \mathrm{~N}_{3} \mathrm{~S}$ & 155 & 50 & $68.44(68.14)$ & $4.09(4.14)$ & $7.48(7.40)$ & $5.70(5.74)$ \\
3d & $\mathrm{C}_{31} \mathrm{H}_{21} \mathrm{O}_{4} \mathrm{~N}_{3} \mathrm{SBr}$ & 201 & 45 & $61.03(61.45)$ & $3.44(3.54)$ & $6.89(6.80)$ & $5.25(5.28)$ \\
3e & $\mathrm{C}_{31} \mathrm{H}_{21} \mathrm{O}_{4} \mathrm{~N}_{3} \mathrm{SCl}$ & $\mathrm{Oily}$ & 53 & $65.78(65.70)$ & $3.71(3.65)$ & $7.42(7.36)$ & $5.65(5.61)$ \\
3f & $\mathrm{C}_{31} \mathrm{H}_{21} \mathrm{O}_{6} \mathrm{~N}_{4} \mathrm{~S}$ & 213 & 40 & $64.58(64.51)$ & $3.64(3.73)$ & $9.72(9.65)$ & $5.55(5.50)$ \\
\hline
\end{tabular}


Table 2. Spectral analysis of title compounds

\begin{tabular}{|c|c|c|c|}
\hline Compd & $\mathrm{IR}\left(\mathrm{KBr}, \mathrm{cm}^{-1}\right)$ & $\begin{array}{c}{ }^{1} \mathrm{H} \mathrm{NMR}\left(\mathrm{CDCl}_{3}{ } \mathrm{DMMSO}-\mathrm{d}_{6}\right), \\
(\delta \mathrm{ppm})\end{array}$ & $\begin{array}{c}{ }^{3} \mathrm{C} \mathrm{NMR}\left(\mathrm{CDCl}_{3} \backslash \mathrm{DMSO}-\mathrm{d}_{6}\right) \\
(\delta \mathrm{ppm})\end{array}$ \\
\hline $2 \mathbf{a}$ & $\begin{array}{l}3325(\mathrm{~N}-\mathrm{H}), \\
3040(\mathrm{Ar}-\mathrm{H}), \\
1580(\mathrm{C}=\mathrm{N}), \\
2920(\mathrm{C}-\mathrm{H}), \\
1320 \& 1140\left(\mathrm{SO}_{2}\right)\end{array}$ & $\begin{array}{l}3.30\left(2 \mathrm{H}, \mathrm{t}, \mathrm{CH}_{2} \mathrm{~N}=\right), 3.00(2 \mathrm{H}, \mathrm{t}, \\
\left.\mathrm{CH}_{2} \mathrm{NH}\right), 8.75(1 \mathrm{H}, \mathrm{s}, \mathrm{NHC}=\mathrm{C}, \\
\left.\text { inte.0.62),6.02( } \mathrm{OCH}_{2} \mathrm{O}\right), \\
\text { 7.01(1H,>CH, inte.0.42), } \\
\text { 7.15-8.45 (14H,aromatic })\end{array}$ & $\begin{array}{l}\text { 120-150(twenty one lines, } \\
\text { 21aromatic carbon), } 164 \& 165 \\
\text { (two lines,two } \mathrm{C}=\mathrm{N}) \text {, } \\
\text { 99 }\left(\mathrm{OCH}_{2} \mathrm{O}\right), 45 \& 47(\mathrm{two} \\
\left.\text { lines, }=\mathrm{N}-\mathrm{CH}_{2} \mathrm{CH}_{2}-\mathrm{NH}\right), 83(>\mathrm{CH})\end{array}$ \\
\hline $2 \mathbf{b}$ & $\begin{array}{l}3320(\mathrm{~N}-\mathrm{H}), \\
3050(\mathrm{Ar}-\mathrm{H}), \\
1590(\mathrm{C}=\mathrm{N}), \\
2924(\mathrm{C}-\mathrm{H}), \\
1324 \& 1135\left(\mathrm{SO}_{2}\right)\end{array}$ & $\begin{array}{l}3.37\left(2 \mathrm{H}, \mathrm{t}, \mathrm{CH}_{2} \mathrm{~N}=\right), 3.15(2 \mathrm{H}, \mathrm{t}, \mathrm{C} \\
\left.\mathrm{H}_{2} \mathrm{NH}\right), 8.69(1 \mathrm{H}, \mathrm{s}, \mathrm{NHC}=\mathrm{C} \text {,inte. } \\
0.62), 5.94\left(\mathrm{OCH}_{2} \mathrm{O}\right), 7.00(1 \mathrm{H},> \\
\mathrm{CH}, \text { inte.0.44),7.25-8.54 } \\
(13 \mathrm{H}, \text { aromatic }), 2.35\left(3 \mathrm{H}, \mathrm{s}, \mathrm{CH}_{3}\right)\end{array}$ & $\begin{array}{l}\text { 118-152(twenty one lines, } \\
\text { 21aromatic carbon), } 162 \& 164 \\
\text { (two lines, two C=N) } \\
99\left(\mathrm{OCH}_{2} \mathrm{O}\right), 46 \& 48 \text { (two lines, } \\
\left.=\mathrm{N}_{-} \mathrm{CH}_{2} \mathrm{CH}_{2}-\mathrm{NH}\right) \\
84(>\mathrm{CH}) 21\left(\mathrm{CH}_{3}\right)\end{array}$ \\
\hline $2 c$ & $\begin{array}{l}3322(\mathrm{~N}-\mathrm{H}), \\
3060(\mathrm{Ar}-\mathrm{H}), \\
1585(\mathrm{C}=\mathrm{N}), \\
2940(\mathrm{C}-\mathrm{H}), \\
1327 \& 1130\left(\mathrm{SO}_{2}\right)\end{array}$ & 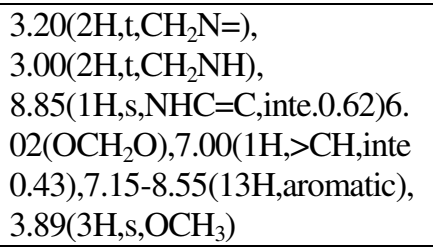 & $\begin{array}{l}115-150(\text { twenty one lines, } \\
21 \text { aromatic carbon), } 164 \& \\
166(\text { two lines, two } \mathrm{C}=\mathrm{N}) \\
100\left(\mathrm{OCH}_{2} \mathrm{O}\right), 46 \& 47(\text { two lines } \\
\left.=\mathrm{N}-\mathrm{CH}_{2} \mathrm{CH}_{2}-\mathrm{NH}\right), \\
84(>\mathrm{CH}) 56\left(0 \mathrm{CH}_{3}\right)\end{array}$ \\
\hline $2 d$ & $\begin{array}{l}3325(\mathrm{~N}-\mathrm{H}), \\
3056(\mathrm{Ar}-\mathrm{H}), \\
1594(\mathrm{C}=\mathrm{N}), \\
2930(\mathrm{C}-\mathrm{H}), \\
1315 \& 1120\left(\mathrm{SO}_{2}\right)\end{array}$ & $\begin{array}{l}\left.\text { 3.32(2H,t, } \mathrm{CH}_{2} \mathrm{~N}=\right), \\
2.98\left(2 \mathrm{H}, \mathrm{t}, \mathrm{CH}_{2} \mathrm{NH}\right), 8.70 \\
(1 \mathrm{H}, \mathrm{s}, \mathrm{NHC}=\mathrm{C}, \text { inte. } 0.62), \\
\left.\text { 6.05( } \mathrm{OCH}_{2} \mathrm{O}\right), \\
\text { 7.14(1H,>CH,inte.0.38), } \\
\text { 7.25-8.45(13H,aromatic) }\end{array}$ & $\begin{array}{l}\text { 120-152(twenty one lines, } \\
21 \text { aromatic carbon), } \\
163 \& 165(\text { two lines, twoC }=\mathrm{N}), \\
100\left(\mathrm{OCH}_{2} \mathrm{O}\right), 45 \& 46(\text { two lines } \\
\left.=\mathrm{N}-\mathrm{CH}_{2}-\mathrm{CH}_{2}-\mathrm{NH}\right), 83(>\mathrm{CH})\end{array}$ \\
\hline $2 e$ & $\begin{array}{l}3335(\mathrm{~N}-\mathrm{H}), \\
3052(\mathrm{Ar}-\mathrm{H}), \\
1584(\mathrm{C}=\mathrm{N}), \\
2910(\mathrm{C}-\mathrm{H}), \\
1318 \& 1130\left(\mathrm{SO}_{2}\right)\end{array}$ & 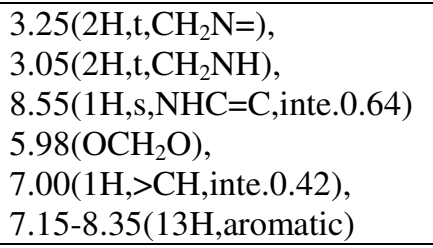 & $\begin{array}{l}\text { 117-156(twenty one lines, } \\
21 \text { aromatic carbon), } \\
164 \& 165(\text { two lines,twoC=N), } \\
100.01\left(\mathrm{OCH}_{2} \mathrm{O}\right), 45 \& 47(\text { two } \\
\left.\text { lines, }=\mathrm{N}-\mathrm{CH}_{2}-\mathrm{CH}_{2}-\mathrm{NH}\right), \\
84(>\mathrm{CH})\end{array}$ \\
\hline $2 f$ & $\begin{array}{l}3325(\mathrm{NH}), \\
3030(\mathrm{Ar}-\mathrm{H}), \\
1588(\mathrm{C}=\mathrm{N}), \\
2928(\mathrm{C}-\mathrm{H}), \\
1326 \& 1137\left(\mathrm{SO}_{2}\right)\end{array}$ & 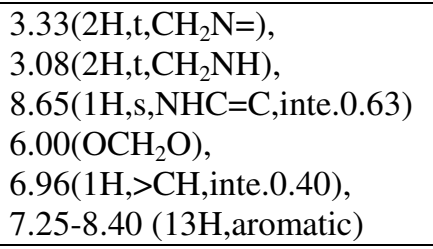 & $\begin{array}{l}\text { 118-155(twenty one lines, } \\
\text { 21aromatic carbon), } 162 \& \\
\text { 164(two lines,twoC=N), } \\
\left.\text { 99.97( } \mathrm{OCH}_{2} \mathrm{O}\right), 43 \& 44(\text { two lines, } \\
\left.=\mathrm{N}-\mathrm{CH}_{2}-\mathrm{CH}_{2}-\mathrm{NH}\right), 82(>\mathrm{CH})\end{array}$ \\
\hline $3 \mathbf{a}$ & $\begin{array}{l}3030(\mathrm{Ar}-\mathrm{H}) \\
1590(\mathrm{C}=\mathrm{N}) \\
2930(\mathrm{C}-\mathrm{H}), \\
3320(\mathrm{~N}-\mathrm{H}), \\
1325 \& 1127\left(\mathrm{SO}_{2}\right)\end{array}$ & $\begin{array}{l}\left.\text { 6.00(2H,s, } \mathrm{OCH}_{2} \mathrm{O}\right), \\
8.65(1 \mathrm{H}, \mathrm{s}, \mathrm{NH}, \text { inte. } 0.80) \\
7.2(1 \mathrm{H}, \mathrm{s},>\mathrm{CH}, \text { inte. } 0.27), \\
7.34-8.53(18 \mathrm{H}, \text { aromatic })\end{array}$ & $\begin{array}{l}\text { 118-153(twenty seven lines, } \\
27 \text { aromatic carbons), } \\
162 \& 165(\text { two lines,two } \mathrm{C}=\mathrm{N}) \text {, } \\
98\left(\mathrm{OCH}_{2} \mathrm{OCH}_{2}\right), 45 \& 47 \\
\left(=\mathrm{NCH}_{2} \mathrm{CH}_{2} \mathrm{NH}\right), 83(>\mathrm{CH})\end{array}$ \\
\hline $3 \mathbf{b}$ & $\begin{array}{l}3035(\mathrm{Ar}-\mathrm{H}) \\
1593(\mathrm{C}=\mathrm{N}) \\
2938(\mathrm{C}-\mathrm{H}) \\
3315(\mathrm{~N}-\mathrm{H}), \\
1315 \& 1120\left(\mathrm{SO}_{2}\right)\end{array}$ & $\begin{array}{l}\left.\text { 6.03(2H,s, } \mathrm{OCH}_{2} \mathrm{O}\right), \\
\text { 8.70(1H,s, NH,inte.0.81) } \\
\text { 7.15(1H,s,>CH,inte.0.25), } \\
\text { 7.24-8.49(17H,aromatic }), \\
\left.\text { 2.25(3H,s, } \mathrm{CH}_{3}\right)\end{array}$ & $\begin{array}{l}119-154 \text { (twenty seven lines, } 27 \\
\text { aromatic carbons), } \\
162 \& 163(\text { two lines, two } \mathrm{C}=\mathrm{N}) \text {, } \\
100\left(\mathrm{OCH}_{2} \mathrm{OCH}_{2}\right), 45 \& 46 \\
\left(=\mathrm{NCH}_{2} \mathrm{CH}_{2} \mathrm{NH}\right), 83(>\mathrm{CH}) \\
22\left(\mathrm{CH}_{3}\right)\end{array}$ \\
\hline
\end{tabular}




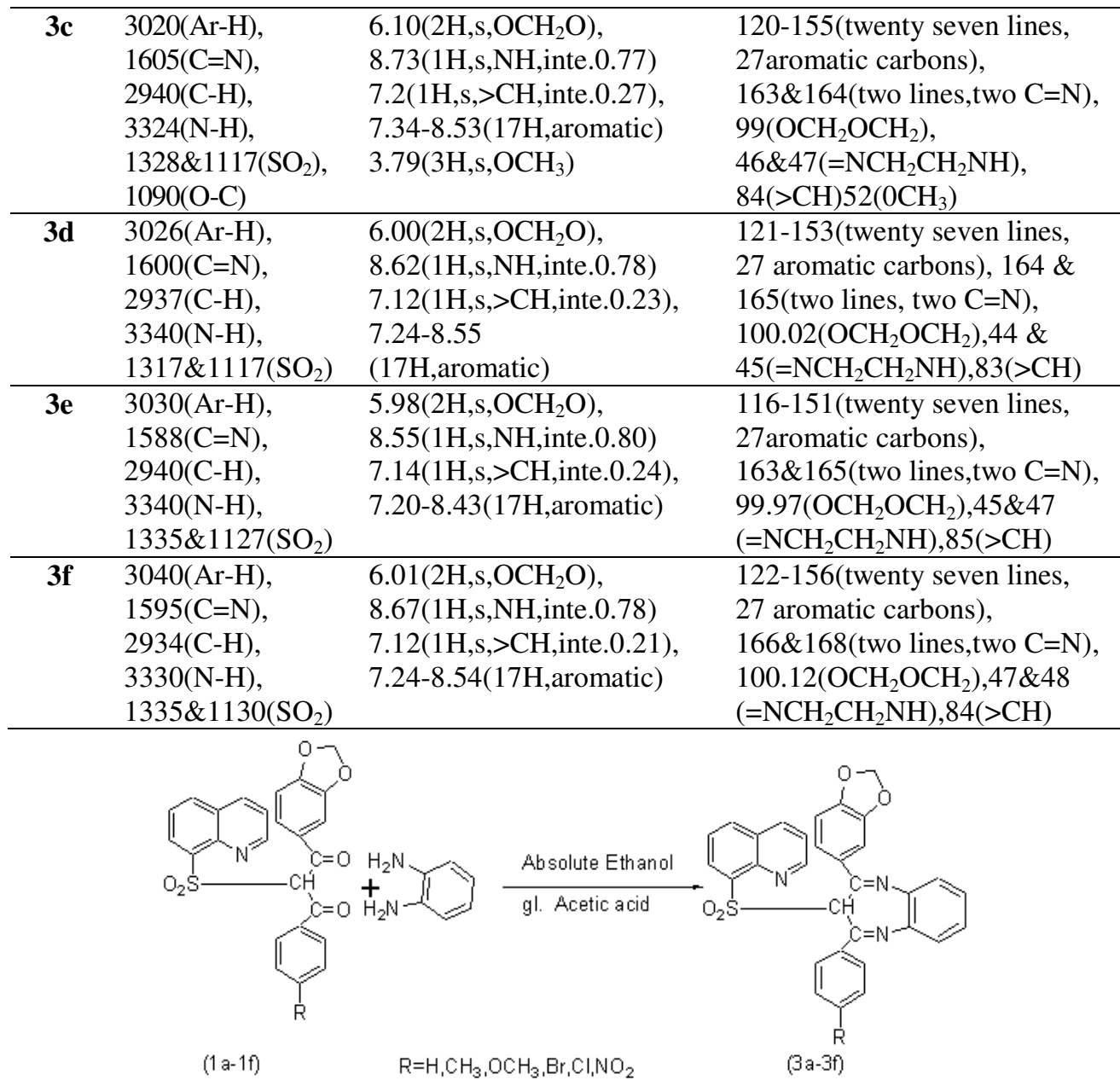

Scheme 2

Tautomerization forms also exist in diazepines and benzodiazepines. On the behalf of integration of $\mathrm{NH}$ proton and active $\mathrm{CH}$ proton, we have established that $\mathrm{A} \&(\mathrm{~B}+\mathrm{C})$ forms are exist in 4:6 ratio. Integration values of $\mathrm{NH} \& \mathrm{CH}$ protons are given in ${ }^{1} \mathrm{H}$ NMR data of Table 2.

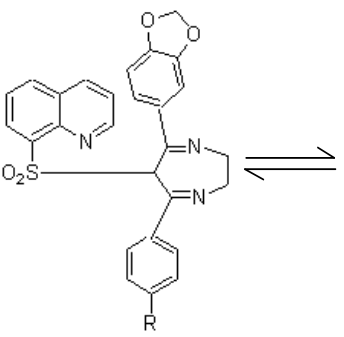

(A)

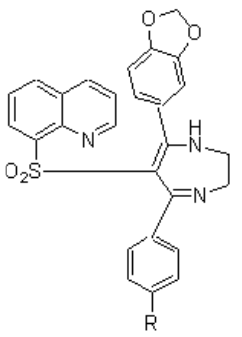

(B)

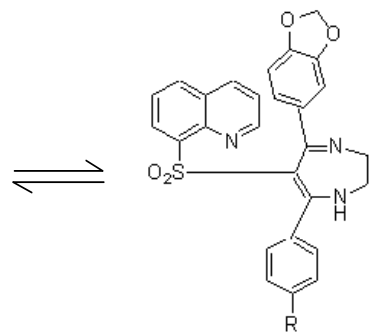

(C)

Scheme 3. Tautomerization in 1, 4-diazepines 
Similarly on the behalf of integration of $\mathrm{NH}$ proton and active $\mathrm{CH}$ proton, we have established that $\mathrm{D} \&(\mathrm{E}+\mathrm{F})$ forms are existing in 2.5:7.5 ratios. Integration values of $\mathrm{NH} \&$ $\mathrm{CH}$ protons are given in ${ }^{1} \mathrm{H}$ NMR data of Table 2.

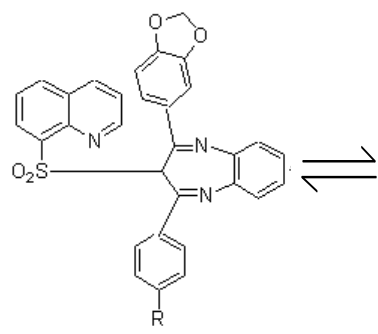

(D)

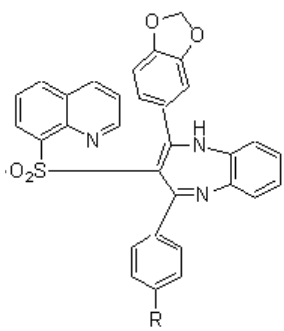

(E)

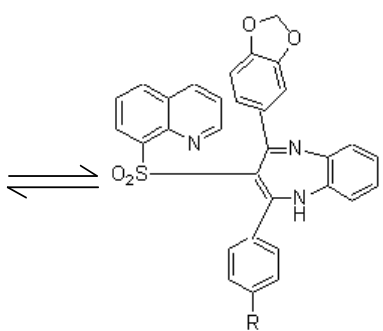

(F)

Scheme 4.Tautomerization in 1, 5-benzodiazepine

\section{Spectral studies}

The IR spectra of compounds (2a-2f, 3a-3f) showed a NH stretching vibration around $3320 \mathrm{~cm}^{-1}$, Ar-H stretching vibration around $3040 \mathrm{~cm}^{-1}, \mathrm{C}-\mathrm{H}$ stretching vibration around $2940 \mathrm{~cm}^{-1}$ and $\mathrm{SO}_{2}$ stretching vibrations at $1140 \& 1320 \mathrm{~cm}^{-1}$ respectively.

The ${ }^{1} \mathrm{H}$ NMR spectra of compounds (2a-2f, 3a-3f) showed a multiplet in the range of $\delta 6.75-8.50 \mathrm{ppm}$ due to aromatic protons. A singlet observed around $\delta 6.01 \mathrm{ppm}$ is due to presence of dioxymethylene proton, a singlet observed at $\delta 8.75 \mathrm{ppm}$ is due to presence of $\mathrm{NH}$ proton and triplet observed around $\delta 3.15 \mathrm{ppm}$ is due to presence of $-\mathrm{NCH}_{2} \mathrm{CH}_{2} \mathrm{~N}$ - protons.

The ${ }^{13} \mathrm{C}$ NMR spectra of compounds (2a-2f) showed 21 lines between region $\delta 120-150 \mathrm{ppm}$ due to aromatic carbon. Two carbon of $\mathrm{C}=\mathrm{N}$ groups are appeared around $\delta 165 \mathrm{ppm}$. The carbon of dioxymethylene group appeared at $\delta 100 \mathrm{ppm}$. Two lines for two carbons of $\mathrm{CH}_{2}$ attached to nitrogen atoms of diazepine ring appeared around $\delta 47 \mathrm{ppm}$. Active methylene carbon which appeared in diketones at $\delta 88.13 \mathrm{ppm}$ undergo slightly upward shift and observed at $885 \mathrm{ppm}$ indicating the formation of diazepine ring.

The ${ }^{13} \mathrm{C}$ NMR spectra of compounds (3a-3f) showed 27 lines between region $\delta 115-152 \mathrm{ppm}$ due to aromatic carbons. Two carbon of $\mathrm{C}=\mathrm{N}$ groups are appeared around $\delta 162 \& 165 \mathrm{ppm}$. The carbon of dioxymethylene group appeared at $\delta 101 \mathrm{ppm}$. Two lines for two carbons of $\mathrm{CH}_{2}$ attached to nitrogen atoms of diazepine ring appeared around $\delta 47 \& \delta 44 \mathrm{ppm}$. Active methylene carbon which appeared in diketones at $\delta 88.13 \mathrm{ppm}$ undergo slightly upward shift and observed at $\delta 83 \mathrm{ppm}$ indicating the formation of diazepine nucleus.

\section{Acknowledgement}

Authors are thankful to Head, Department of Chemistry, University of Rajasthan, Jaipur for providing laboratory facilities. One of them (Rajendra Kumar Saini) is thankful to the CSIR, New Delhi for the award of Junior Research Fellowship.

\section{References}

1. Krezel I, Mikiciuk Olasik E, Zurek E and Glowka M L, Pharm Pharmacol Commun., 1999, 5, 458.

2. Antoine M, Barreau M, Desconclois J F, Girard P and Picaut G, Eur Pat Appl EP, 379 412/1990; Chem Abstr., 1991, 114, 143393p.

3. Hirokawa Y, Harada H, Yoshikawa T, Yoshida N and Kato S, Chem Pharm Bull., 2002, 50(7), 941-959. 
4. Phillips O A, Murthy K S K, Fiakpui C Y and Knaus E.E, Can J Chem., 1999, 77(2), 216-222.

5. Vicentini C B, Guarneri M, Scatturin A, Giori P and Heilman W, Farmaco, 1996, 51, 609.

6. Stokbroekx R A, Van der Aa MJ M, Luyckx M G M and Grauwels G A J, Chem Abstra., 1990, 112, 35876y.

7. Kurmeier H A, Weber W D, Randunz H E and Schliep H J, Chem Abstr., 1990, 113, 6370 u.

8. Ninomiya K, Nitta K, Tobe A, Egawa M and Kikumoto R, Chem Abstr., 1987, 107, 59050x.

9. Peduto V, Chem Abstr., 2002, 136, 350033.

10. Tonegawa T and Nozaki Y, Chem Abstr., 1999, 131, 252566u.

11. Fourrier A, Letenneur L, Dartigues J F, Moore N and Degaud B, Eur J Clinical Pharma., 2001, 57(5), 419-425.

12. Toshiyuki K, Hiroshi T, Koreichi K, Hideki H, Kohji S, Masafumi A, Tetsuya Fujimoto and Iwao Yamamoto, J Heterocycle Chem., 2002, 39(1), 163-171.

13. Beasley C M, Tollefson G, Tran P, Satterlee W, Sanger T and Hamilton S, Neuropsychopharmacology, 1996, 14(2), 111-123.

14. Thruston D E, Neidle S and Waring M J, The MacMillan Press Ltd., London, 1993, 54.

15. Kamal A, Laxman N, Ramesh G, Neelima K and Kondapi A K, Chem Commun., 2001, 437.

16. Gregson S J, Howard P W, Hartley J A, Brooks N A, Adams L J, Terence C J, Kelland L R and Thruston D E, J Med Chem., 2001, 44(5), 737-748.

17. Unny R, Joshi P and Dhobal M P and Joshi Y C, Heterocycl Commun., 2003, 9, 171. 